\title{
SOME REGULARITY THEOREMS FOR TYPICALLY REAL FUNCTIONS ${ }^{1}$
}

\author{
GEORGE B. LEEMAN, JR.
}

Abstract. This paper studies the class of typically real functions and the subclass of typically real functions with fixed second coefficient. For each of these classes the author proves a few regularity theorems, which describe the coefficients' asymptotic behavior.

Let $S$ be the collection of functions $f(z)=z+\sum_{n=2}^{\infty} a_{n} z^{n}$ analytic and univalent in the unit disk $D=\{z|| z \mid<1\}$. A special case of Hayman's regularity theorem $\left[1\right.$, p. 104] says that if $f(z)=z+\sum_{n=2}^{\infty} a_{n} z^{n} \in S$, then

$$
\lim _{n \rightarrow \infty} \frac{\left|a_{n}\right|}{n}=\tau \text { exists and } \tau \leqq 1,
$$

with equality holding if and only if $f(z)=z /\left(1-e^{i \theta} z\right)^{2}$ for some real $\theta$. See also Milin [6] for a neat proof of this fact. In this paper we shall prove analogous results for the class $T$ of typically real functions.

A function $f(z)=z+\sum_{k=2}^{\infty} a_{k} z^{k}$ analytic in $D$ is said to be typically real provided $f(z)$ is real if and only if $z$ is real. If $-2 \leqq c \leqq 2$, we denote by $T(c)$ the collection of all functions $f(z)=z+c z^{2}+\sum_{k=3}^{\infty} a_{k} z^{k} \in T$. Rogosinski [8], [9] introduced the class $T$ and established many of its important properties. Robertson [7] used his results to show that each function in $T$ has a Stieltjes integral representation of the form

$$
f(z)=z+\sum_{k=2}^{\infty} a_{k} z^{k}=\int_{0}^{\pi} \frac{z}{1-2 z \cos \theta+z^{2}} d \alpha(\theta),
$$

where $\alpha$ is nondecreasing in $[0, \pi]$ and $\alpha(0)=0, \alpha(\pi)=1$. A computation shows that $z /\left(1-2 z \cos \theta+z^{2}\right)=\sum_{k=1}^{\infty}(\sin k \theta / \sin \theta) z^{k}$, and hence

$$
a_{k}=\int_{0}^{\pi} \frac{\sin k \theta}{\sin \theta} d \alpha(\theta), \quad k=2,3, \cdots .
$$

Received by the editors July 28, 1972 and, in revised form, November 17, 1972.

AMS (MOS) subject classifications (1970). Primary 30A40; Secondary 30A34.

Key words and phrases. Typically real functions, regularity theorems, constrained extremal problems.

${ }^{1}$ This paper describes a portion of the author's doctoral thesis written under the direction of Professor Peter L. Duren at the University of Michigan. 
In particular, any $f \in T$ has real coefficients, and

if $f \in T(c)$.

$$
\int_{0}^{\pi} \cos \theta d \alpha(\theta)=\frac{c}{2}
$$

The statement in (1) is false for functions in $T$. Indeed, the function

$$
f(z)=z+\sum_{n=2}^{\infty} a_{n} z^{n}=\frac{1}{2} \frac{z}{(1-z)^{2}}+\frac{1}{2} \frac{z}{(1+z)^{2}}=\sum_{n=1}^{\infty}(2 n-1) z^{2 n-1}
$$

is in $T$, but the $\operatorname{limits}_{\lim _{n \rightarrow \infty}} a_{n} / n, \lim _{n \rightarrow \infty}\left|a_{n}\right| / n$ clearly fail to exist. However, we can prove some modified regularity theorems. Let us remark that the existence of any limit appearing in each of our results is part of the conclusion.

THEOREM 1. If $f(z)=z+\sum_{k=2}^{\infty} a_{k} z^{k} \in T$, then

$$
-1 \leqq \lim _{n \rightarrow \infty} \frac{a_{2 n}}{2 n} \leqq 1, \quad 0 \leqq \lim _{n \rightarrow \infty} \frac{a_{2 n+1}}{2 n+1} \leqq 1 .
$$

Equality can occur in the first inequality only for the functions $f(z)=$ $z /(1 \pm z)^{2}$. In the second inequality we have equality on the right-hand side only for $f(z)=\lambda\left[z /(1-z)^{2}\right]+(1-\lambda)\left[z /(1+z)^{2}\right]$, where $0 \leqq \lambda \leqq 1$. Equality holds on the left-hand side unless $f(z)=\lambda\left[z /(1 \pm z)^{2}\right]+(1-\lambda) g(z)$, where $0<\lambda \leqq 1$ and $g \in T$.

Proof. Put

$$
\begin{aligned}
& u_{e}(\theta)=1 \quad \text { if } \theta=0, \\
& =0 \quad \text { if } 0<\theta<\pi \text {, } \\
& =-1 \text { if } \theta=\pi \text {; } \\
& \begin{aligned}
u_{0}(\theta)=1 & \text { if } 0=0, \\
=0 & \text { if } 0<0<\pi, \\
=1 & \text { if } \theta=\pi .
\end{aligned}
\end{aligned}
$$

Pick $\alpha$ so that (2) holds; then (3) implies that

so that

$$
\frac{a_{2 n}}{2 n}=\int_{0}^{\pi} \frac{1}{2 n} \frac{\sin 2 n \theta}{\sin \theta} d \alpha(\theta)
$$

$$
\lim _{n \rightarrow \infty} \frac{a_{2 n}}{2 n}=\int_{0}^{\pi} u_{e}(\theta) d \alpha(\theta)
$$

by Lebesgue's dominated convergence theorem. The statements of equality follow easily from the definition of $u_{e}$. Similarly,

$$
\lim _{n \rightarrow \infty} \frac{a_{2 n+1}}{2 n+1}=\int_{0}^{\pi} u_{0}(\theta) d \alpha(\theta)
$$

with the indicated cases of equality. Consequently, Theorem 1 follows. 
COROLLARY 1. If $f \in T$, then $0 \leqq \lim _{r \rightarrow 1-}(1-r)^{2} f(r) \leqq 1$. Equality holds on the left unless $f(z)=\lambda\left[z /(1-z)^{2}\right]+(1-\lambda) g(z)$, where $0<\lambda \leqq 1$ and $g \in T$. Equality holds on the right only for $f(z)=z /(1-z)^{2}$.

Proof. We shall use the well-known fact (see for example [3, pp. 490-491]) that the Cesàro summability of a sequence implies its Abel summability to the same value. Let $f(z)=z+\sum_{k=2}^{\infty} a_{k} z^{k}$, and put

$$
\begin{gathered}
L_{c}=\lim _{n \rightarrow \infty} \frac{a_{2 n}}{2 n}, \quad L_{0}=\lim _{n \rightarrow \infty} \frac{a_{2 n+1}}{2 n+1}, \\
a_{k}=0 \quad \text { if } k \leqq 0, \\
b_{k}=\frac{1}{2}\left(a_{2 k}-2 a_{2 k-2}+a_{2 k-4}\right), \quad k=1,2, \cdots, \\
h(r)=\sum_{k=1}^{\infty} b_{k} r^{k}, \quad-1<r<1 .
\end{gathered}
$$

Then the sequence $\left\{b_{k}\right\}_{k=1}^{\infty}$ is Cesàro-summable to $L_{e}$. Therefore it is Abel-summable, and we deduce that $\lim _{r \rightarrow 1-}(1-r)^{2}[f(r)+f(-r)]=L_{e}$. Using the same procedure for the odd coefficients, we arrive at $\lim _{r \rightarrow 1-}(1-r)^{2}[f(r)-f(-r)]=L_{0}$. Consequently,

$$
\lim _{r \rightarrow 1-}(1-r)^{2} f(r)=\frac{L_{e}+L_{0}}{2}, \quad \lim _{r \rightarrow 1-}(1-r)^{2} f(-r)=\frac{L_{e}-L_{0}}{2} .
$$

If we choose $\alpha$ so that (2) holds, then (5) and (6) yield $\frac{1}{2}\left(L_{e}+L_{0}\right)=$ $\int_{0}^{\pi} \frac{1}{2}\left(u_{e}(\theta)+u_{0}(\theta)\right) d \alpha(\theta)$. The desired inequality and statements of equality easily follow from the definitions of $u_{e}$ and $u_{0}$.

For any function $f$ continuous in $D$, let us define $M_{\infty}(r, f)=$ $\max _{|z|=r}|f(z)|$ for each $r<1$. Hayman showed that if $f \in S$, then $\lim _{r \rightarrow 1-}(1-r)^{2} M_{\infty}(r, f)=\tau$, where $\tau$ is as in (1). We shall prove a related result for the class $T$.

Suppose $g(z)=1+\sum_{k=1}^{\infty} b_{k} z^{k}$ is analytic in $D$ and $\operatorname{Re} g(z)>0$ there. We need two well-known facts about $g$ :

$$
M_{\infty}(r, g) \leqq(1+r) /(1-r), \quad 0 \leqq r<1,
$$

and

$$
\left|b_{k}\right| \leqq 2, \quad k=1,2, \cdots .
$$

Equality holds in (7) or $\left|b_{1}\right|=2$ in (8) only if $g(z)=\left(1+e^{i \theta} z\right) /\left(1-e^{i \theta} z\right)$ for some real $\theta$. Both of these results follow from the Schwarz lemma. To prove (7), let $h(z)=(1+z) /(1-z)$ and apply the lemma to $h^{-1}[g(z)]$. To prove (8) for a fixed value of $k$, let $t_{1}, \cdots, t_{k}$ be all the $k$ th roots of unity, 
and apply the lemma to $h^{-1}[f(z)]$, where

$$
f(z)=\frac{1}{k} \sum_{j=1}^{k} g\left(t_{j} z^{1 / k}\right)=1+\sum_{l=1}^{\infty} b_{l}\left(\frac{1}{k} \sum_{j=1}^{k} t_{j}^{l}\right) z^{l / k}=1+\sum_{l=1}^{\infty} b_{l l} z^{l} .
$$

LEMma 1. If $f \in T$, then $\left|f^{\prime}(z)\right| /|f(z)| \leqq(1+|z|) /|z|(1-|z|)$ for all $z \in D-\{0\}$. If equality holds for a fixed $z \neq 0$, then equality holds for all $z$, and $f(z)=z /(1 \pm z)^{2}$.

Proof. Rogosinski [9] has shown that if $f \in T$, then

$$
g(z)=\left(\left(1-z^{2}\right) / z\right) f(z)
$$

has positive real part in $D$. Fix $z_{0} \in D-\{0\}$, and apply (8) to

to arrive at

$$
\begin{aligned}
h(z) & =\frac{1}{\operatorname{Re} g\left(z_{0}\right)}\left[g\left(\frac{z+z_{0}}{1+\bar{z}_{0} z}\right)-i \operatorname{Im} g\left(z_{0}\right)\right] \\
& =1+\frac{g^{\prime}\left(z_{0}\right)\left(1-\left|z_{0}\right|^{2}\right)}{\operatorname{Re} g\left(z_{0}\right)} z+\cdots
\end{aligned}
$$

$$
\left|\frac{g^{\prime}\left(z_{0}\right)}{g\left(z_{0}\right)}\right| \leqq \frac{\left|g^{\prime}\left(z_{0}\right)\right|}{\operatorname{Re} g\left(z_{0}\right)} \leqq \frac{2}{1-\left|z_{0}\right|^{2}} .
$$

If equality holds throughout (10), then we can use the statement following (8) and the fact that $g$ has real coefficients to write

$$
g(z)=g\left(z_{0}\right) \frac{\left(e^{i \theta}-\bar{z}_{0}\right) z+\left(1-e^{i \theta} z_{0}\right)}{-\left(e^{i \theta}+\bar{z}_{0}\right) z+\left(1+e^{i \theta} z_{0}\right)}=g\left(z_{0}\right) \frac{a z+b}{c z+d},
$$

where $a, b, c, d$ are real and

$$
\frac{a}{c}=-\frac{e^{i \theta}-\bar{z}_{0}}{e^{i \theta}+\bar{z}_{0}}=-\left(\frac{1-e^{i \theta} z_{0}}{1+e^{i \theta} z_{0}}\right)=-\frac{\bar{b}}{\bar{d}}=-\frac{b}{d} .
$$

However, $g(0)=1$; thus $g(z)=(-c z+d) /(c z+d)=1-2 c z / d+\cdots$, whence $g(z)=(1 \pm z) /(1 \mp z)$. Lemma 1 now follows from (9), (10) and what we have just shown.

THEOREM 2. If $f \in T$, then $\lim _{r \rightarrow 1-}(1-r)^{2} M_{\infty}(r, f) \leqq 1$. Equality holds only for $f(z)=z /(1 \pm z)^{2}$.

Proof. In view of Lemma 1, the proof is identical to [1, pp. 8-10]. We shall now solve two of the preceding problems for the class $T(c)$.

THEOREM 3. If $f \in T(c)$, then $0 \leqq \lim _{r \rightarrow 1-}(1-r)^{2} f(r) \leqq(2+c) / 4$. Equality 
holds on the right only for

$$
f(z)=\frac{2+c}{4} \frac{z}{(1-z)^{2}}+\frac{2-c}{4} \frac{z}{(1+z)^{2}} ;
$$

equality holds on the left unless

$$
f(z)=\frac{2 c-g^{\prime \prime}(0)}{4-g^{\prime \prime}(0)} \frac{z}{(1-z)^{2}}+\frac{4-2 c}{4-g^{\prime \prime}(0)} g(z),
$$

where $g \in T$ and $g^{\prime \prime}(0) \neq 2 c$.

Proof. Jenkins [2] showed that if $f \in T(c)$, then

$$
\frac{r}{1-c r+r^{2}} \leqq f(r) \leqq \frac{2+c}{4} \frac{r}{(1-r)^{2}}+\frac{2-c}{4} \frac{r}{(1+r)^{2}},
$$

with equality on the left or right only for $f(z)=z /\left(1-c z+z^{2}\right)$ or $f(z)=$ $\frac{1}{4}(2+c) z /(1-z)^{2}+\frac{1}{4}(2-c) z /(1+z)^{2}$, respectively. Our assertions easily follow from this result and Corollary 1.

We next prove a constrained version of Theorem 1 . Note that the results of [4] yield the crude estimate $0.196 c-0.608 \leqq \lim _{n \rightarrow \infty} a_{2 n} / 2 n \leqq 0.196 c+$ 0.608 for a function $f(z)=z+c z^{2}+\sum_{k=3}^{\infty} a_{k} z^{k} \in T(c)$. The correct coefficient of $c$ is not 0.196 , but 0.250 . The theorem which follows, however, is most remarkable in that the best bounds cannot in general be attained.

THEOREM 4. Let $f(z)=z+c z^{2}+\sum_{k=3}^{\infty} a_{k} z^{k} \in T(c)$. Then

and

$$
\frac{c-2}{4} \leqq \lim _{n \rightarrow \infty} \frac{a_{2 n}}{2 n} \leqq \frac{c+2}{4}
$$

$$
0 \leqq \lim _{n \rightarrow \infty} \frac{a_{2 n+1}}{2 n+1} \leqq 1 .
$$

The constants in (11) are best possible, but equality can hold only when $c= \pm 2$. In (12) equality holds on the right only for

$$
f(z)=\frac{2+c}{4} \frac{z}{(1-z)^{2}}+\frac{2-c}{4} \frac{z}{(1+z)^{2}} .
$$

Equality holds on the left unless

$$
f(z)=\frac{g^{\prime \prime}(0)-2 c}{g^{\prime \prime}(0) \pm 4} \frac{z}{(1 \pm z)^{2}}+\frac{2 c \pm 4}{g^{\prime \prime}(0) \pm 4} g(z),
$$

where $g \in T$ and $g^{\prime \prime}(0) \neq 2 c$. 
Proof. The second inequality (12) and cases of equality follow from Theorem 1. For each $k, k=1,2, \cdots$, set

$$
\begin{aligned}
u_{k}(\theta) & =1-k \theta & & \text { if } 0 \leqq \theta \leqq 1 / k, \\
& =0 & & \text { if } 1 / k \leqq \theta \leqq \pi-1 / k, \\
& =k(\pi-\theta)-1 & & \text { if } \pi-1 / k \leqq \theta \leqq \pi,
\end{aligned}
$$

and define a functional $\Phi_{k}$ on $T(c)$ by setting $\Phi_{k}(f)=\int_{0}^{\pi} u_{k}(\theta) d \alpha(\theta)$, where $\alpha$ represents $f$ as in (2). To attain the minimum or maximum value of $\Phi_{k}(f)$, we may choose $\alpha$ to be a step-function with at most two discontinuities $\theta_{1}, \theta_{2}, 0 \leqq \theta_{1} \leqq \theta_{2} \leqq \pi$, by Theorem 3 of [5]. In other words, by (4) we may assume that $f$ is of the form

$$
f(z)=\frac{c-t}{s-t} \frac{z}{1-s z+z^{2}}+\frac{s-c}{s-t} \frac{z}{1-t z+z^{2}},
$$

where $s=2 \cos \theta_{2}, t=2 \cos \theta_{1}$, and $-2 \leqq s \leqq c \leqq t \leqq 2$. A computation yields

$$
\begin{aligned}
\int_{0}^{\pi} u_{k}(\theta) d \alpha(\theta) & =0 & & \text { if } s>-2, t<2, \\
& =c / 2 & & \text { if } s=-2, t=2, \\
& =(c-t) /(2+t) & & \text { if } s=-2, t<2, \\
& =(c-s) /(2-s) & & \text { if } s>-2, t=2,
\end{aligned}
$$

provided $k$ is sufficiently large. Now $\sup _{-2<s \leqq c}(c-s) /(2-s)=(c+2) / 4$, $\inf _{c \leqq t<2}(c-t) /(2+t)=(c-2) / 4$, thus letting $k \rightarrow \infty$ and applying dominated convergence produces $(c-2) / 4 \leqq \int_{0}^{\pi} u_{e}(\theta) d \alpha(\theta) \leqq(c+2) / 4$. Furthermore, the computations above show that these bounds are best possible; consequently, (11) now follows from (5).

Next, if $c=2$, then $f(z)=z /(1-z)^{2}$, and equality holds in (11). Now fix $c<2$, and suppose there exists an $f \in T(c)$, represented by $\alpha$ as in (2), for which

$$
\int_{0}^{\pi} u_{e}(\theta) d \alpha(\theta)=\alpha(0+0)+\alpha(\pi-0)-1=\frac{c+2}{4} .
$$

We claim that $\alpha$ cannot be a step-function. Assume on the contrary that we can find points $0=\theta_{0}<\theta_{1}<\cdots<\theta_{n}<\theta_{n+1}=\pi$ and constants $0=$ $\lambda_{0} \leqq \lambda_{1} \leqq \cdots \leqq \lambda_{n+1} \leqq \lambda_{n+2}=1$ such that $\alpha(\theta)=\lambda_{k}$ when $\theta_{k-1}<\theta<\theta_{k}$, $k=1, \cdots, n+1$. We cannot have $n=0$, for in that case

$$
f(z)=z+c z^{2}+\sum_{k=3}^{\infty} a_{k} z^{k}=\frac{2+c}{4} \frac{z}{(1-z)^{2}}+\frac{2-c}{4} \frac{z}{(1+z)^{2}},
$$


and $\lim _{n \rightarrow \infty} a_{2 n} / 2 n=c / 2<(c+2) / 4$, a contradiction; hence $n \geqq 1$, and $\alpha$ has at least one discontinuity in $(0, \pi)$. Now (13) yields $\lambda_{1}, \lambda_{n+1} \geqq(c+2) / 4$; therefore

$$
\lambda_{n+1}-\lambda_{1} \leqq(2-c) / 4 .
$$

Using (4) and (13) gives

$$
\left(\lambda_{n+1}-\lambda_{1}\right) \cos \theta_{n} \leqq \sum_{k=2}^{n+1}\left(\lambda_{k}-\lambda_{k-1}\right) \cos \theta_{k-1}=\frac{c-2}{4},
$$

and therefore $\theta_{n}=\pi$, by (14). This contradiction establishes our claim that $\alpha$ cannot be a step-function. As a consequence we can find points $0<\theta_{1}<\theta_{2}<\pi$ such that

$$
\alpha(0+0)<\alpha\left(\theta_{1}-0\right) \leqq \alpha\left(\theta_{1}+0\right)<\alpha\left(\theta_{2}\right) .
$$

Fix $\theta_{3}$ with $\theta_{2}<\theta_{3}<\pi$, and put

$$
\beta(\theta)=\chi_{\theta_{1} \theta_{3}}\left[\alpha\left(\theta_{1}+0\right)-\alpha(\theta)\right], \quad \gamma(\theta)=\chi_{0 \theta_{1}}\left[\alpha\left(\theta_{1}-0\right)-\alpha(\theta)\right],
$$

where $\chi_{a b}$ denotes the characteristic function on the open interval $(a, b)$. Integration by parts yields

$$
\begin{aligned}
& p=\int_{0}^{\pi} \cos \theta d \beta(\theta)=\int_{0}^{\pi} \beta(\theta) \sin \theta d \theta \leqq 0, \\
& q=\int_{0}^{\pi} \cos \theta d \gamma(\theta)=\int_{0}^{\pi} \gamma(\theta) \sin \theta d \theta \geqq 0,
\end{aligned}
$$

and we can choose $r>0$ so that

$$
1+p r>0, \quad 1-q r>0 .
$$

Define $\alpha_{*}(\theta)=\alpha(\theta)+r[q \beta(\theta)-p \gamma(\theta)]$; then $\alpha_{*}$ is nondecreasing on $[0, \pi]$ by (17), and (16) shows that (4) holds. Thus $\alpha_{*}$ represents a function in $T(c)$, and we conclude that

$$
\alpha_{*}(0+0)+\alpha_{*}(\pi-0)-1 \leqq \alpha(0+0)+\alpha(\pi-0)-1,
$$

that is, $r p\left[\alpha\left(\theta_{1}-0\right)-\alpha(0+0)\right] \geqq 0$. However, (15) shows that $\alpha\left(\theta_{1}-0\right)-$ $\alpha(0+0)>0$, and $p<0$, because $\beta$ is negative on $\left(\theta_{2}, \theta_{3}\right)$. This final contradiction shows that equality cannot hold on the right-hand side of (11) unless $c=2$. The case of equality on the left-hand side follows if we replace $f(z)$ by $g(z)=-f(-z)$. We have now completed the proof of Theorem 4.

To gain more insight into the discontinuous behavior of the functional appearing in Theorem 4 , assume $c \neq \pm 2$, and choose a sequence $\left\{s_{m}\right\}_{m=1}^{\infty}$ such that $-2<s_{m}<c, m=1,2, \cdots, \lim _{m \rightarrow \infty} s_{m}=-2$. Let

$$
f(z)=\frac{2+c}{4} \frac{z}{(1-z)^{2}}+\frac{2-c}{4} \frac{z}{(1+z)^{2}}=z+c z^{2}+\sum_{k=3}^{\infty} a_{k} z^{k}
$$


and

$$
\begin{array}{r}
f_{m}(z)=\frac{c-s_{m}}{2-s_{m}} \frac{z}{(1-z)^{2}}+\frac{2-c}{2-s_{m}} \frac{z}{1-s_{m} z+z^{2}}=z+c z^{2}+\sum_{k=3}^{\infty} a_{m k} z^{k} \\
m=1,2, \cdots .
\end{array}
$$

Then

$$
\lim _{m \rightarrow \infty} \lim _{n \rightarrow \infty} \frac{a_{m, 2 n}}{2 n}=\frac{c+2}{4}, \quad \lim _{n \rightarrow \infty} \lim _{m \rightarrow \infty} \frac{a_{m, 2 n}}{2 n}=\frac{c}{2},
$$

and we see that interchanging the limit operations yields the same answer only when $c=2$.

\section{REFERENCES}

1. W. K. Hayman, Multivalent functions, Cambridge Tracts in Math. and Math. Phys., no. 48, Cambridge Univ. Press, Cambridge, 1958. MR 21 \#7302.

2. J. A. Jenkins, Some problems for typically real functions, Canad. J. Math. 13 (1961), 299-304. MR 22 \#12227.

3. K. Knopp, Theory and application of infinite series, Hafner, New York, 1947.

4. G. B. Leeman, The constrained coefficient problem for typically real functions, Trans. Amer. Math. Soc. (to appear).

5. - Constrained extremal problems for families of Stieltjes integrals (to appear).

6. I. M. Milin, Hayman's regularity theorem for the coefficients of univalent functions, Dokl. Akad. Nauk SSSR 192 (1970), 738-741 =Soviet Math. Dokl. 11 (1970), 724-728. MR 42 \#3269.

7. M. S. Robertson, On the coefficients of a typically-real function, Bull. Amer. Math. Soc. 41 (1935), 565-572.

8. W. Rogosinski, Über positive harmonische Sinusentwicklungen, Jber. Deutsch. Math.-Verein. 40 (1931), 33-35.

9. —— Über positive harmonische Entwicklungen und typisch-reelle Potenzreihen, Math. Z. 35 (1932), 93-121.

IBM Thomas J. Watson Research Center, Yorktown Heights, New York 10598 